\title{
Digital pathology - implementation challenges in low-resource countries
}

\author{
Paul Fontelo ${ }^{\mathrm{a}, *}$, John Faustorilla ${ }^{\mathrm{b}}$, Alex Gavino ${ }^{\mathrm{a}, \mathrm{b}}$ and Alvin Marcelo ${ }^{\mathrm{b}}$ \\ ${ }^{a}$ National Library of Medicine, Bethesda, MA, USA \\ ${ }^{\mathrm{b}}$ National Telehealth Center, University of the Philippines Manila, Manila, Philippines
}

\begin{abstract}
Background: Medical education in pathology and histology in low-resource countries face many obstacles because of equipment cost and telecommunication deficiencies. Digital Pathology may provide solutions. We report student experience with virtual slides on a local network and a remote image server.

Methods: Using an iPad tablet device, fifty 3rd and 4th year medical students viewed digital pathology slides from a Web server at the National Library of Medicine and a mirror server on the local network.

Results: The quality of images from both servers was found to be satisfactory, but the local server was deemed faster and preferred by the participants in this study $(p<0.005)$.

Conclusions: Virtual slides on a local network server may provide solutions to equipment and technical obstacles and could enhance student learning in developing countries.
\end{abstract}

Keywords: Digital pathology, virtual slide, virtual microscope, whole slide imaging, medical education, low-resource countries

\section{Introduction}

Teaching histology and pathology - image intensive subjects that require microscopes and glass slides - in medical, nursing and allied health professional schools in developing countries is confronted with many challenges. Among them, the high cost of acquisition and maintenance of microscopes. As a consequence, two or more students may have to share a microscope in the teaching laboratory (Fig. 1). Furthermore, glass slide specimens may also be shared among students since preparation of new specimens and replacement of broken slides are often expensive as well. Moreover, the time for laboratory hours is limited, and microscopes are often locked up and unavailable for use

*Corresponding author: Paul Fontelo, MD, MPH, National Library of Medicine 8600, Rockville Pike, Bethesda, MD 20894, USA. Tel.: +1 301435 3265; Fax: +1 301402 4080; E-mail: fontelo@nlm.nih.gov. after class hours. Finally, the faculty-to-student ratio is also frequently higher due to lack of pathologists in academia.

Digital pathology, already in use in many medical schools in the United States and other developed countries, has been shown to provide advantages over the usual method of teaching histology and pathology [1-4]. In developing countries, digital pathology is practiced in the form of telepathology in clinical practice [5-9]. The use of digital pathology (telepathology, teledermatology) in education [10], second-opinion consultations [11-14] and primary diagnosis [15-18] has been reported in several journals. In digital pathology, an entire microscopic glass slide (virtual slide, whole slide imaging) is captured with a digital camera attached to a microscope, converted to an electronic format then archived on a Web server that can be viewed on a computer monitor with a Web browser. In some settings, plug-ins or special browsers may be required. It avoids the requirement of using micro- 


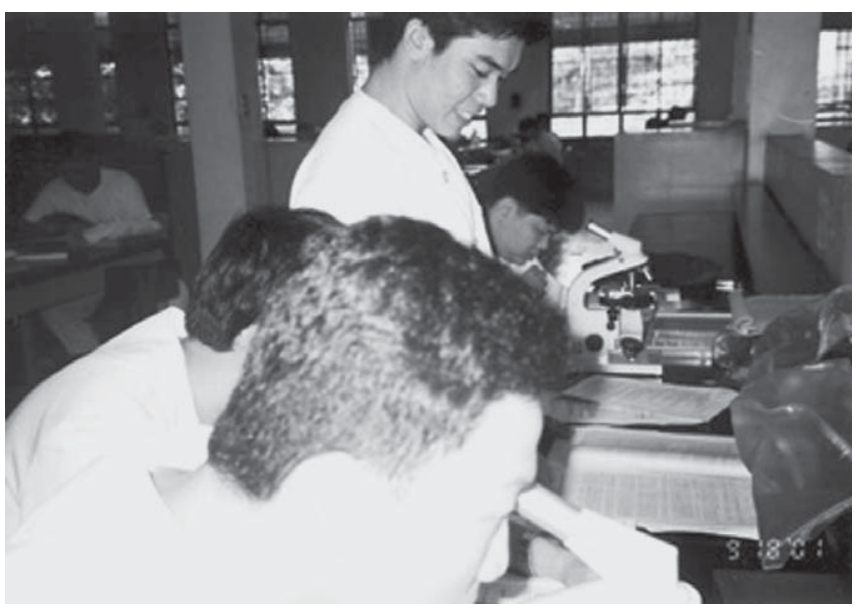

Fig. 1. Medical students in a pathology laboratory sharing microscopes and slides.

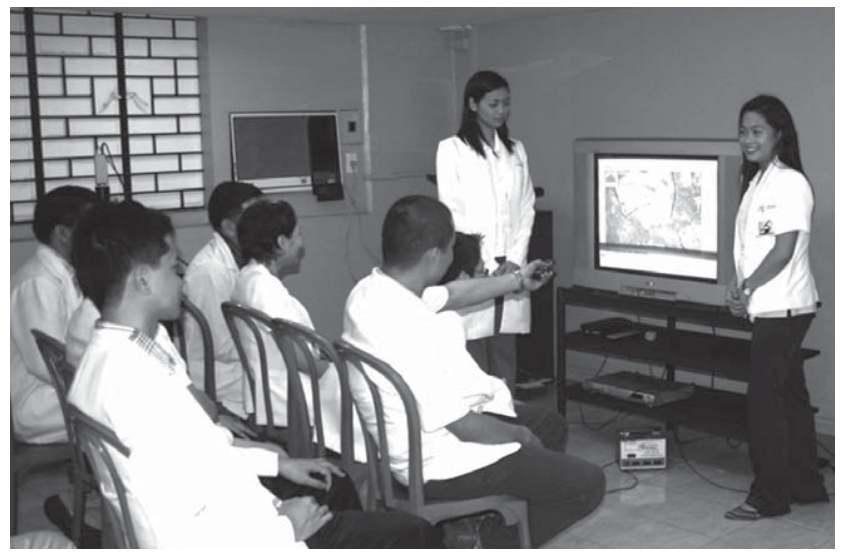

Fig. 2. With digital pathology, teaching, discussion, and student and faculty interaction is enhanced.

scopes and glass slides while providing the feature of viewing the entire slide. The dynamic and interactive mode of viewing images (horizontal and vertical movement of images, and zooming in and out) simulates viewing a glass slide under the microscope (virtual microscope). Its interactive features and facility to view the image on a large monitor promotes group interaction and discussion (Fig. 2). Images can be viewed anytime with an Internet or Intranet connected desktop computer, portable or tablet computer, or even through smartphones anywhere.

Its main challenge may be the requirement for high bandwidth. The high bandwidth constraint became apparent in a previous study to evaluate the accuracy of diagnosis of virtual slides [19]. Some participants traveling in remote areas outside of the United States had to rely on dialup connections to view the slides. Frequent timeouts and poor quality images made it immediately apparent that viewing virtual slides on low bandwidth, dialup links was not possible.

Implementing digital pathology in low-resource areas remains a challenge even today. Access to the Internet on academic networks is often slow and expensive. Other barriers include: the high cost of equipment for digitizing glass slides and limited student access to computer workstations especially after class hours. In developing countries, electrical power may be unreliable, outages frequent, and adverse weather events could disrupt telecommunications. Methods to alleviate some of these drawbacks are discussed below.

Pathologists' attitude towards digital pathology is mixed [20-25]. In remote locations where there a lack of trained pathologists exists, the use of real-time, dynamic telepathology through whole slide imaging is 
Table 1

Participants' responses comparing NLM (US) and UPCM (local) server based on a 5-point Likert scale, 1-Strongly disagree, 5-Strongly agree

\begin{tabular}{lccc}
\hline Indicators & $\begin{array}{c}\text { NLM server } \\
\text { (US) }\end{array}$ & $\begin{array}{c}\text { Local server } \\
\text { (UPCM) }\end{array}$ & $p$-value \\
\hline The site is easy to access using an iPad & 4.04 & 4.62 & 0.0001 \\
The site is easy to navigate using an iPad & 3.82 & 4.5 & 0.0001 \\
The site loads fast enough to display a clear image & 3.86 & 4.52 & 0.0003 \\
I see myself using this site to address my academic needs & 3.98 & 4.42 & 0.0048 \\
\hline
\end{tabular}

increasing. Nevertheless, we are not aware of studies that explore the student perspective of digital pathology especially in developing countries. In this paper, we attempt to determine the student's attitudes in one medical school towards digital pathology.

\section{Methods}

Teaching glass slides were provided by the Department of Pathology, Uniformed Services University [26]. The images were scanned using an Aperio T3 Scanscope, then, using Zoomifyer EZ the images were tiled and segmented into smaller JPG files suitable for downloading on the Web. A copy of the Web archive of virtual slides (18 gigabytes) from the US National Library of Medicine (NLM) was transported on a portable hard drive then copied to the local Web server in the University of the Philippines College of Medicine (UPCM) in Manila, Philippines. Both servers use an Apache server, PHP, and MySQL. The images were configured for display on Apple iOS devices (iPhone, iPod and iPad) using BrainMapsCASK API [27] from Leiden University Medical Center.

Fifty medical students $(\mathrm{MSIII}=3, \mathrm{MSIV}=47$; male $=24$, female $=26$; age range, 20-29 years, mode $=23$ years) from the school were recruited during enrollment time to participate in a non-randomized study. The number of students who did not participate was unknown. All of the participants had already passed the required Pathology course in their second year. After verbal consent was obtained, they were instructed to view two bookmarked identical Web sites, one at the National Library of Medicine (NLM) and the other, a local mirror server at UPCM (http://images.nlm.nih.gov/pathlab9. and http://zell.telehealth.ph/pathlab respectively) The participants used a first generation iPad (Apple) tablet computer and its default Safari Web browser. The iPad
Table 2

Medical students' preferences comparing NLM (US) and UPCM (local) server

\begin{tabular}{lcc}
\hline Indicators & $\begin{array}{c}\text { NLM server } \\
\text { (US) }\end{array}$ & $\begin{array}{c}\text { Local server } \\
\text { (UPCM) }\end{array}$ \\
\hline Quality of images using an iPad & 6 & 44 \\
Ease of navigation using an iPad & 6 & 44 \\
Faster image loading & 10 & 40 \\
Usage in addressing academic needs & 9 & 41 \\
\hline
\end{tabular}

connected to the medical school's shared wireless network with average tested speeds of 300 Kilobits/sec download and 54 Kilobits/sec upload. Both Websites were configured for iOS devices that did not require Adobe Flash player, unlike the usual Web site (http://images.nlm.nih.gov/pathlab) that did. After randomly reviewing images from both Websites lasting between 5-10 minutes, they were asked to complete a paper-based evaluation. They rated their experience based on their perception of ease of navigation, access speed, and preferences comparing the digitized images from Web server at the NLM and UPCM servers on a 5-point Likert scale (1-Strongly Disagree, 2-Disagree, 3-Somewhat agree, 4-agree, 5-Strongly Agree). Comments were encouraged but not required. No personally identifiable information was collected.

\section{Results}

Table 1 shows that the participating medical students rated the local UPCM as significantly faster and responsive to their school needs. The participants also overwhelmingly preferred the local server to the NLM server in all questions (Table 2).

Although not required, all of the students provided written comments to almost all of the questions posed. Their observations generally concurred that the images from the local server loaded faster and of the almost seamless quality in viewing the slides displayed. These 
reflected their ratings shown in Tables 1 and 2. They also validated one of virtual slides' significant features - that virtual slides simulate the experience of examining glass slides under an optical microscope.

Some general comments common to both servers were: it "makes patho[logy] studies interesting", "high quality pictures", "simple interface", "the pictures are very nice and the site is more aesthetically pleasing", "interactive". The slides were labeled with the diagnosis only, so many comments talked about, "no labels of structures, lack of descriptions" and that they would find it more useful and easier for navigation if parts of the slides have labels, rather than just having diagnosis.

The specific comments on the servers reflect the bandwidth deficiency that slowed the display of digital images from the NLM server. They found the local server to be "simple", "fast loading", "zooming, loading time in local server is faster than US server", and "local server is faster!" Conversely, some described the digital images from US server were described as having "choppy transitions", "a bit slower than the local one", and "harder to zoom in and out".

\section{Discussion}

Although access to the Internet in the Philippines is widespread with $33 \%$ of the population connected to the Internet [28], bandwidth in most places is still less than $1 \mathrm{Mb} / \mathrm{s}$. Metropolitan areas may attain speeds greater than $1 \mathrm{Mb} / \mathrm{s}$ but this may not be the case in most other locations in the country. In this study, the network in the medical school, which is situated at the nation's capital, is rated at $8 \mathrm{Mb} / \mathrm{s}$ but desktop and wireless access distributed across the campus is still less than $1 \mathrm{Mb} / \mathrm{s}$.

The participants in this study accessed digital slides archived in a local server and in a Web server through the Internet. Although the medical students found their experience to be generally satisfactory in this study, digital pathology implementation in developing countries may still be limited by slow connections to the Internet. However, slow connectivity or unavailability of Internet access need not hamper the use of digital pathology in developing countries. In conducting this study we discovered that digital slides stored on a portable hard drive or USB drive can be connected directly to a local computer and displayed in a Web browser. In situations where several students need access simultaneously, a local machine may be used as the server in an Intranet setup. The increasing capacity and lowering costs of these portable storage devices make them suitable alternatives to accessing virtual slides through the Internet. At present, connecting portable hard drives to mobile handheld devices, such as a tablet computer, is not yet generally feasible. However, mobile devices can use microSD (secure digital) memory cards, which might enable this process.

The use of digital slides in medical education makes the study of histology and pathology a meaningful yet interesting endeavor for students [29]. In this study, we received positive comments that showed how students perceived the use of digital pathology in medical school. The students also suggested that they would find useful and easier to navigate if parts of the slides had labels, rather than just having diagnosis. This is a feature that has been suggested in the past so the next version of this collection will likely include labels to diagnostic features of the slide. An option will be provided to switch between labeled and unlabeled versions. Essential instructional and navigational features of slides could be added to thumbnail images to aid the student but which could be hidden when not needed. The unlabeled images might be useful for instructors and for student assessment.

In developed countries, local area networks are often assumed to be robust and adequately managed. However, it does not usually prevail in low-to-medium income countries where reliability is a continuing challenge. On top of the expense of network hardware, there is also the cost of hiring and retaining competent technical personnel to install, configure, and keep the local network running at optimal levels. Although local access to the digital slides received better reviews from the students in this study, the hidden cost of keeping a reliable network should be considered when planning the appropriate placement of servers and wireless networks.

Since the study was done in a single medical school in the Philippines - it may not be universally applicable. However, since many of the conditions mentioned earlier prevail in developing countries, the lessons learned in this study might provide suggestions on the implementation of digital pathology in low-resource areas. The non-randomized and non-blinded nature of this study is a limitation. More systematic, randomized, and blinded studies are indicated. Another limitation of the study is the use of a single iPad by the students. With simultaneous, multi-user use access, the 
local area network may become congested if it is not configured properly to balance the load across high demand mobile devices. Quality of service may suffer and the teacher/student experience will be less than optimal.

Although a local archive and an external drive might be adequate for viewing slides locally, many academic organizations provide virtual slide archives that might be useful for student teaching and resident training. Future studies might also include experiments to determine the minimum acceptable bandwidth for viewing virtual slides. Perhaps, a reassessment of pathologists' attitudes and acceptance of telepathology and whole slide imaging is needed. In regions of the world where there are not enough pathologists, it is a necessity. The cost of scanning slides is still prohibitive in most developing countries. Collaboration between academic centers in developed countries with digital scanning equipment and universities in developing countries with teaching slides that may be rare and unusual in developed countries, could enhance medical education for all. Ultimately, it may be cost-effective for a university to send their teaching slides to partner institutions by mail or courier who can digitize them for sharing across participating schools.

\section{Conclusion}

Our results show that access to both servers was satisfactory but the local server was deemed faster and preferred by a majority of participants in this study. Virtual slides, accessible through a local server or portable drive may be a solution to the high bandwidth requirement of digital pathology or in places where the Internet in unavailable. Collaborations with universities in developed countries would enhance image collections for teaching for institutions and these can be shared with others.

\section{Acknowledgments}

Figure 1. Photo courtesy of Dr. Kirk Patrick Uy.

Figure 2. Photo courtesy of Dr. Siegfried Perez; published in "Development of a Virtual Slide Library in a Developing Country". Encinas MA, Ducut E, Liu F, Avila JM, Diwa M, Fontelo P. AMIA Annu Symp Proc; 2009; 836.

\section{References}

[1] R.A. Bloodgood and R.W. Ogilvie, Trends in histology laboratory teaching in United States medical schools, Anat Rec B New Anat 289 (2006), 169-175.

[2] F. Dee and P. Heidger, Virtual slides for teaching histology and pathology, in: Virtual Microscopy and Virtual Slides in Teaching, Diagnosis, and Research, in Taylor \& Francis Group, CRC Press, Boca Raton, (2005), 151-159.

[3] B.B. Krippendorf and J. Lough, Complete and rapid switch from light microscopy to virtual microscopy for teaching medical histology, The Anatomical Record Part B: The New Anatomist 285B (2005), 19-25.

[4] F.P. Paulsen, M. Eichhorn and L. Bräuer, Virtual microscopyThe future of teaching histology in the medical curriculum? Annals of Anatomy - Anatomischer Anzeiger 192 (2010) 378-382.

[5] E. Ayad and F. Sicurello, Telepathology in emerging countries pilot project between Italy and Egypt, Diagn Pathol 3(1) (2008), S2.

[6] K. Brauchli, R. Jagilly, H. Oberli, K.D. Kunze, G. Phillips, N. Hurwitz, et al., Telepathology on the Solomon Islandstwo years' experience with a hybrid Web- and email-based telepathology system, J Telemed Telecare 10 (Suppl 1) (2004), 14-17.

[7] F. Pagni, F. Bono, C. Di Bella, A. Faravelli and A. Cappellini, Virtual surgical pathology in underdeveloped countries: The zambia project, Archives of Pathology \& Laboratory Medicine 135 (2011), 215-219.

[8] H. Góngora Jará and H.A. Barcelo, Telepathology and continuous education: Important tools for pathologists of developing countries, Diagn Pathol 3(1) S24.

[9] F.G. Mullick, P. Fontelo and C. Pemble, Telemedicine and telepathology at the Armed Forces institute of pathology: History and current mission, Telemed J 2 (1996), 187-193.

[10] R.S. Weinstein, The education of professionals, Hum Pathol 34 (2003), 415-416.

[11] S.S.P. Costello, D.J. Johnston, P.A. Dervan and D.G. O'Shea, Development and evaluation of the virtual pathology slide: A new tool in telepathology, J Med Internet Res 5 (2003), e11.

[12] B. Molnar, L. Berczi, C. Diczhazy, A. Tagscherer, S.V. Varga, B. Szende, et al., Digital slide and virtual microscopy based routine and telepathology evaluation of routine gastrointestinal biopsy specimens, J Clin Pathol 56 (2003), 433-438.

[13] D.H. Okada, S.W. Binder, C.L. Felten, J.S. Strauss and A.M. Marchevsky, "Virtual microscopy" and the internet as telepathology consultation tools: Diagnostic accuracy in evaluating melanocytic skin lesions, Am J Dermatopathol 21 (1999), 525-531.

[14] D.M. Steinberg and S.Z. Ali, Application of virtual microscopy in clinical cytopathology, Diagn Cytopathol 25 (2001), 389-396.

[15] A.J. Evans, R. Chetty, B.A. Clarke, S. Croul, D.M. Ghazarian and T-R Kiehl, et al., Primary frozen section diagnosis by robotic microscopy and virtual slide telepathology: The University Health Network experience, Hum Pathol 40 (2009), 1070-1081.

[16] B.E. Dunn, H. Choi, D.L. Recla, S.E. Kerr and B.L. Wagenman, Robotic surgical telepathology between the Iron Mountain and 
Milwaukee Department of Veterans Affairs Medical Centers: A 12-year experience, Hum Pathol 40 (2009), 1092-1099.

[17] Y. Tsuchihashi, T. Takamatsu, Y. Hashimoto, T. Takashima, K. Nakano and S. Fujita, Use of virtual slide system for quick frozen intra-operative telepathology diagnosis in Kyoto, Japan Diagn Pathol 3(1) S6.

[18] B. Têtu, J. Boulanger and C. Houde, [Telepathology project on virtual slides of eastern Quebec: A clinical project carried out in 21 areas], Ann Pathol 30 (2010), 25-27.

[19] P. Fontelo, E. DiNino, K. Johansen, A. Khan and M. Ackerman, Virtual microscopy: Potential applications in medical education and telemedicine in countries with developing, In: System Sciences, 2005. HICSS'05. Proceedings of the 38th Annual Hawaii International Conference on (2005), 153c.

[20] W.M. Bamford, N. Rogers, M. Kassam, J. Rashbass and P.N. Furness, The development and evaluation of the UK national telepathology network, Histopathology 42 (2003), 110-119.

[21] K. Brauchli, H. Oberli, N. Hurwitz, K.-D. Kunze, G. Haroske, G. Jundt, et al., Diagnostic telepathology: Long-term experience of a single institution, Virchows Arch 444 (2004), 403-409.

[22] W.-Y. Liang, C.-Y. Hsu, C.-R. Lai, D.M.-T. Ho and I.J. Chiang, Low-cost telepathology system for intraoperative frozen-section consultation: Our experience and review of the literature, Hum Pathol 39 (2008), 56-62.
[23] T. Mairinger, Acceptance of telepathology in daily practice, Anal Cell Pathol 21 (2000), 135-140.

[24] T. Mairinger, T.T. Netzer, W. Schoner and A. Gschwendtner, Pathologists' attitudes to implementing telepathology, $J$ Telemed Telecare 4 (1998), 41-46.

[25] N. Singh, N. Akbar, C. Sowter, K.G. Lea and C.A. Wells, Telepathology in a routine clinical environment: Implementation and accuracy of diagnosis by robotic microscopy in a one-stop breast clinic, J Pathol 196 (2002), 351-355.

[26] USU, F. Edward. Hébert School of Medicine, Department of Pathology, http://www.usuhs.mil/pat. Access date 08-192011.

[27] Clinical. Anatomical SKills, http://www.lumc.nl/con/3090/ $82525 / 903101130112533 /$ ?setlanguage $=$ English\&setcountry $=$ en. Access date 08-19-2011.

[28] News Release July11 2011, Singaporeans can't get enough of digital media: Nielsen. http://sg.nielsen.com/site/News ReleaseJuly112011.shtml. Access date 08-19-2011.

[29] R.K. Kumar, B. Freeman, G.M. Velan and P.J. De Permentier, Integrating histology and histopathology teaching in practical classes using virtual slides, Anat Rec B New Anat 289 (2006), $128-133$. 


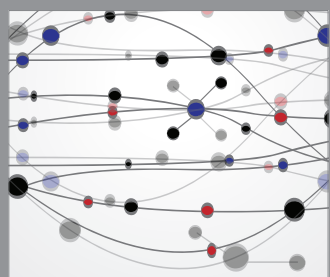

The Scientific World Journal
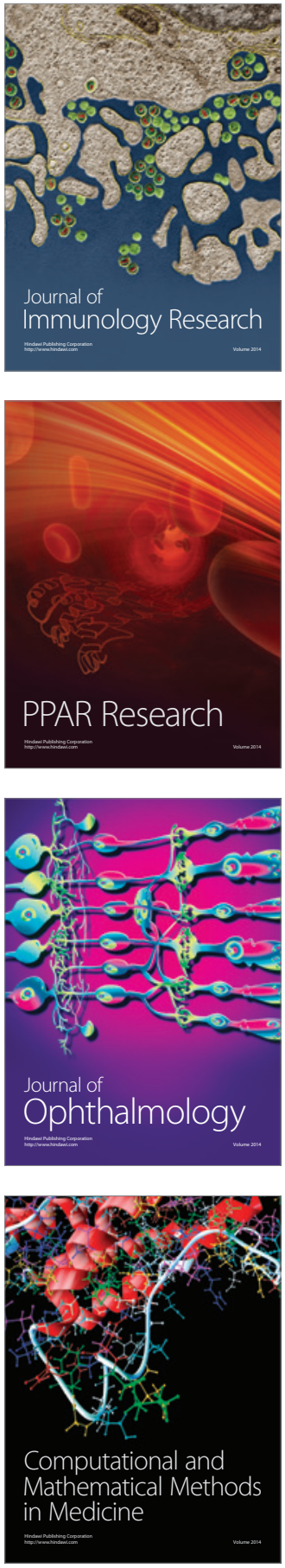

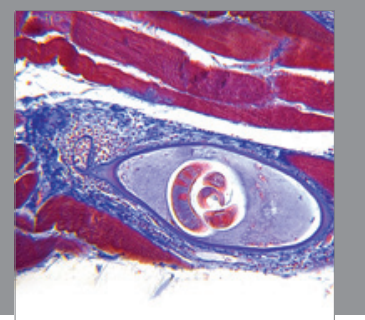

Gastroenterology

Research and Practice
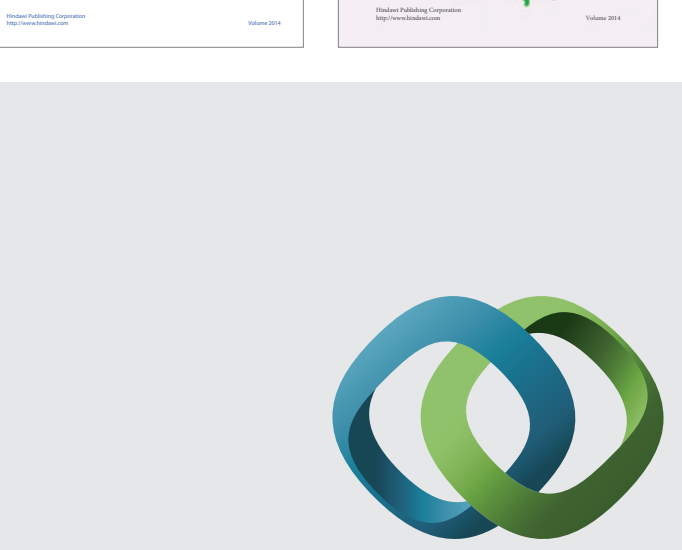

\section{Hindawi}

Submit your manuscripts at

http://www.hindawi.com
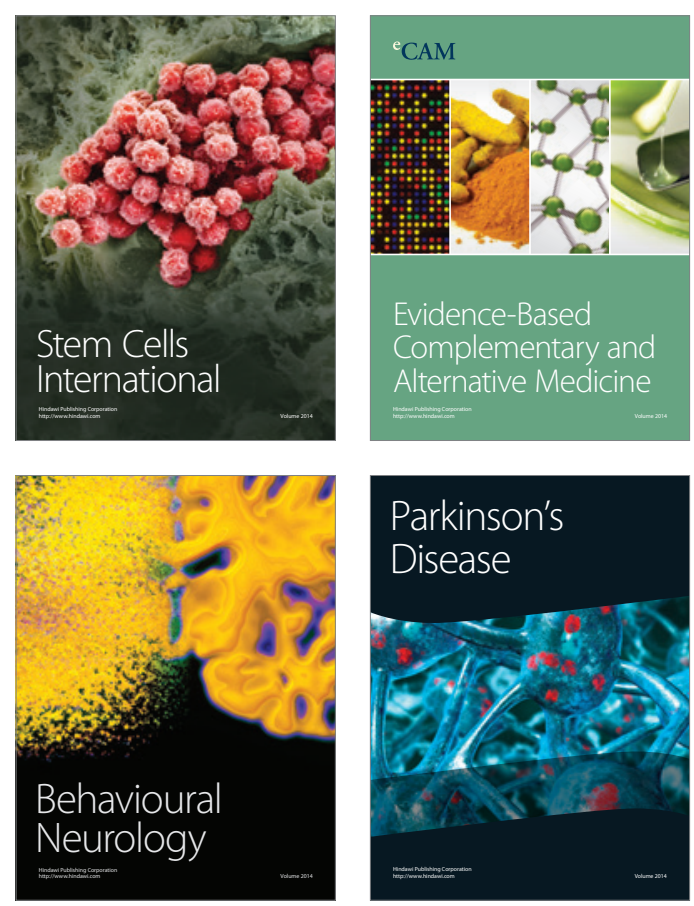

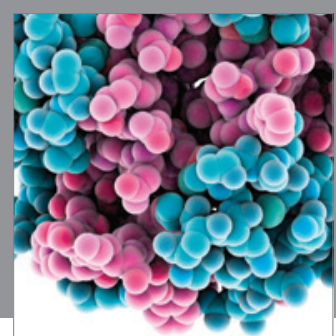

Journal of
Diabetes Research

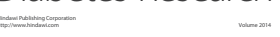

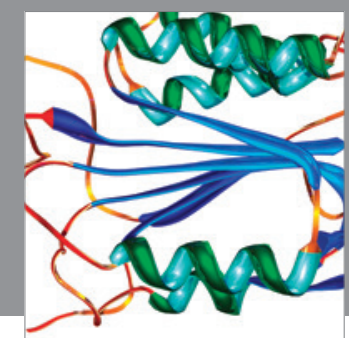

Disease Markers
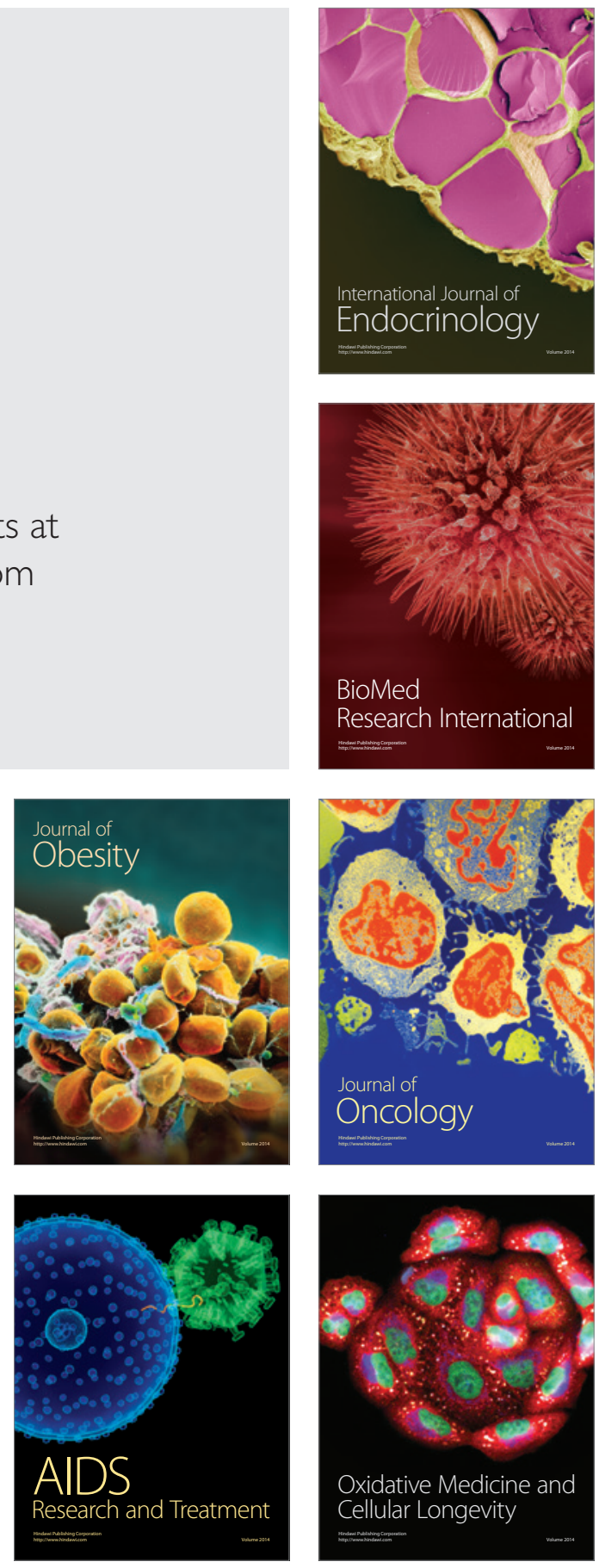